\title{
Effect of Loss on Single Photon Parametric Amplification
}

\author{
Dmitrii N. Vavulin ${ }^{1}$ and Andrey A. Sukhorukov ${ }^{2}$ \\ ${ }^{1}$ ITMO University, 49 Kronverksky Ave., St.Petersburg, 197101, Russia* \\ ${ }^{2}$ Nonlinear Physics Centre, Research School of Physics and Engineering, \\ Australian National University, Canberra, ACT 2601, Australia ${ }^{\dagger}$
}

\begin{abstract}
We analyze the transmission of a single photon through a nonlinear waveguide, under the presence of linear losses and a co-propagating classical pump wave facilitating quantum-injected optical parametric amplification. We derive coupled Schrödinger-type equations which describe the processes of photon loss and generation of photon triplets, and obtain their solution to identify the quantum photon state at the output.

Keywords: Generation of biphotons (Primary), Parametric amplification, Nonlinear waveguides, Quantum state engineering

PACS numbers: 42.65.Tg, 42.65.Lm, 42.25.Bs
\end{abstract}

\section{INTRODUCTION}

A range of quantum protocols, including quantum teleportation [1-4] and quantum cryptography [5-7], can be realized with photons prepared in specific non-classical states. In particular, non-classical photon states can be generated through the process of spontaneous parametric down-conversion (SPDC) in quadratically nonlinear media or spontaneous four-wave-mixing (SFWM) in media with cubic nonlinearity [8-14].

The process of SPDC can be viewed as parametric amplification seeded by vacuum fluctuations. When a quantum photon state is present at the input, the resulting process of quantum-injected optical parametric amplification (QI-OPA) provides a nontrivial state transformation with a variety of applications, see a review [10] and references therein. It was predicted that even a weak input signal can drastically increase the conversion efficiency [15] and just a single input photon can lead to macroscopic differences in the output state [16], which can be used to create micro-macro entanglement or a Schrödinger cat state [17].

Optical losses can have a detrimental effect on photonic quantum states. It was suggested that QI-OPA can be employed to create macro-qubits, which can then fascilitate efficient long range communication over a lossy transmission channel [18]. On the other hand, it was shown that losses inside a nonlinear medium can significantly affect the process of photon-pair generation through SPDC [19, 20] or SFWM [21, 22]. In this work, we perform a detailed theoretical investigation of weak QI-OPA of a single photon, while fully accounting for the effect of linear losses in a nonlinear optical structure.

This paper is organized as follows. In Section II we formulate the theoretical model of single-photon parametric amplification and formulate coupled Schrödinger equations for different photon number states under ap-

\footnotetext{
*dima-vavulin@mail.ru

† Andrey.Sukhorukov@anu.edu.au
}

proximation of a weak pump. In the following Section III we analyse the quantum state evolution and present numerical examples, demonstrating the effect of losses. We present conclusions in Section IV. Appendix A outlines the derivation of Schrödinger equations for the photon state evolution.

\section{MODEL EQUATIONS}

We consider the process of travelling-wave parametric amplification in a quadratically nonlinear waveguide, under the presence of linear losses. We assume narrowband (quasi-cw) pump and photon spectra, and neglect the frequency dispersion effects. Following Ref. [19], the total effective Hamiltonian can be written as:

$$
\hat{H}(z)=\hat{H}_{n l}(z)+\hat{H}_{l o s s}(z) .
$$

Here the first term describes the process of parametric amplification in a lossless waveguide,

$$
\begin{aligned}
\hat{H}_{n l}(z) & =\beta_{s}^{(0)} a_{s}^{\dagger} a_{s}+\beta_{i}^{(0)} a_{i}^{\dagger} a_{i} \\
& +\left[\kappa E_{p}(z) a_{s}^{\dagger} a_{i}^{\dagger}+\kappa E_{p}^{*}(z) a_{s} a_{i}\right],
\end{aligned}
$$

where $z$ is the propagation distance along the waveguide, $a_{s, i}^{\dagger}$ and $a_{s, i}$ are the creation and annihilation operators for the signal and idler photons satisfying the standard commutation relations $\left[a_{s}, a_{s}^{\dagger}\right]=1$ and $\left[a_{i}, a_{i}^{\dagger}\right]=1, \beta_{s}^{(0)}$ and $\beta_{i}^{(0)}$ are the photon propagation constants, $\kappa$ is the effective nonlinear coefficient, $E_{p}$ is the classical pump amplitude, and subscripts 's' and 'i' denote the signal and idler waves, respectively.

To simplify the notations, we define the signal and idler propagation constants relative to half of the pump propagation constant, $\beta_{p} / 2$. Therefore, we formally take $\beta_{p}=0$ in the following, such that the phase mismatch of nonlinear wave-mixing is determined as

$$
\Delta \beta=\beta_{s}+\beta_{i}
$$

Taking into account classical pump attenuation with the 
loss coefficient $\gamma_{p}$, we introduce a normalized pump amplitude $A$ as

$$
\kappa E_{p}(z)=A e^{-\gamma_{p} z} .
$$

The effect of loss can be modelled by the following Hamiltonian [19],

$$
\begin{aligned}
\hat{H}_{\text {loss }}(z) & =\sqrt{2 \gamma_{s}}\left[a_{s} b_{s}^{\dagger}(z)+a_{s}^{\dagger} b_{s}(z)\right] \\
& +\sqrt{2 \gamma_{i}}\left[a_{i} b_{i}^{\dagger}(z)+a_{i}^{\dagger} b_{i}(z)\right],
\end{aligned}
$$

where $\gamma_{s, i}$ are the linear loss coefficients, $b_{s, i}(z)$ are the photon annihilation operators representing the loss at coordinate $z$ with the commutation relations $\left[b_{s}\left(z_{1}\right), b_{s}^{\dagger}\left(z_{2}\right)\right]=\delta\left(z_{1}-z_{2}\right)$ and $\left[b_{i}\left(z_{1}\right), b_{i}^{\dagger}\left(z_{2}\right)\right]=\delta\left(z_{1}-\right.$ $\left.z_{2}\right)$.

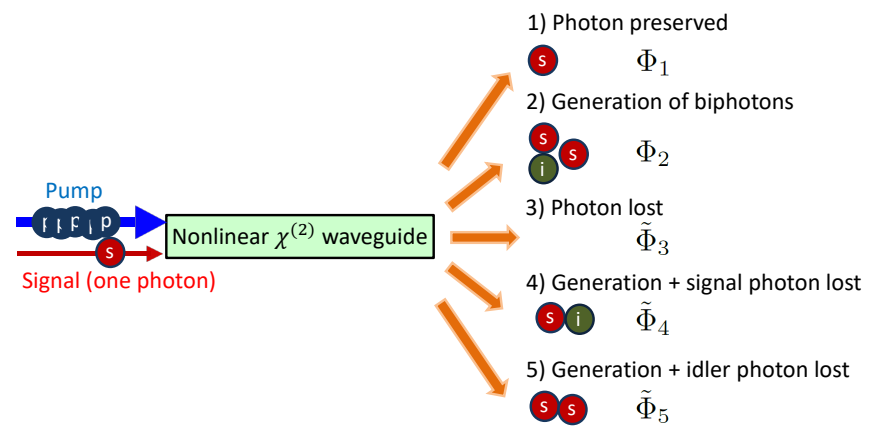

FIG. 1. Scenarios of single input photon propagation through a nonlinear waveguide in the regime of weak parametric amplification and in presence of linear losses.

We now focus on the regime of weak parametric amplification and small losses, when a maximum of one photon pair can be generated. Then three main scenarios can be realized: 1) The signal photon propagates in the waveguide unaffected by nonlinearity and losses; 2) The photon triplet is generated due to the generation of an extra photon pair; 3) The photon is lost. These possibilities are schematically illustrated in Fig. 1 . Then, we seek a solution for a quantum state in the following form, considering specific photon frequencies $\omega_{s}$ and $\omega_{i}$ which can be selected by appropriate excitation and filtering,

$$
\begin{aligned}
|\Psi(z)\rangle & =\Phi_{1}(z) a_{s}^{\dagger}|0\rangle+\Phi_{2}(z) a_{i}^{\dagger} a_{s}^{\dagger} a_{s}^{\dagger}|0\rangle \\
& +\int_{0}^{z} d z_{l} \tilde{\Phi}_{3}\left(z, z_{l}\right) b_{s}^{\dagger}\left(z_{l}\right)|0\rangle \\
& +\int_{0}^{z} d z_{l} \tilde{\Phi}_{4}\left(z, z_{l}\right) a_{i}^{\dagger} b_{s}^{\dagger}\left(z_{l}\right) a_{s}^{\dagger}|0\rangle \\
& +\int_{0}^{z} d z_{l} \tilde{\Phi}_{5}\left(z, z_{l}\right) b_{i}^{\dagger}\left(z_{l}\right) a_{s}^{\dagger} a_{s}^{\dagger}|0\rangle \\
& +\ldots
\end{aligned}
$$

Here $\Phi_{1}$ is the amplitude of a single signal photon, $\Phi_{2}(z)$ corresponds to two signal photons and one idler, $\tilde{\Phi}_{3}\left(z, z_{l}\right)$ to a lost signal photon at a distance $z_{l}, \tilde{\Phi}_{4}\left(z, z_{l}\right)$ to a pair of signal and idler photons and a lost signal photon at a distance $z_{l}, \tilde{\Phi}_{5}\left(z, z_{l}\right)$ to a pair of signal photons and a lost idler photon at a distance $z_{l}$. The other terms are neglected, as they correspond to higher-order cascaded nonlinear processes or multiple lost photons, which by our assumptions have very small probability of occurring.

The evolution of the state vector can be approximately described by the Schrödinger equation for the biphoton wavefunction, where we neglect the vacuum-state dynamics due to higher-order perturbations [19, 23]:

$$
\frac{d|\Psi(z)\rangle}{d z} \approx-i \hat{H}(z)(|0\rangle+|\Psi(z)\rangle) .
$$

We substitute Eq. (6) into Eq. (7), and after collecting various terms in the framework of the perturbation theory (see Appendix A for details), we obtain the following self-consistent equations for the evolution of wavefunction amplitudes:

$$
\begin{aligned}
\frac{\partial \Phi_{1}(z)}{\partial z}= & -\left(i \beta_{s}+\gamma_{s}\right) \Phi_{1}(z) \\
- & i 2 A e^{-\gamma_{p} z} \Phi_{2}(z), \Phi_{1}(0)=1, \\
\frac{\partial \Phi_{2}(z)}{\partial z}= & -\left(i\left(2 \beta_{s}+\beta_{i}\right)+2 \gamma_{s}+\gamma_{i}\right) \Phi_{2}(z) \\
- & i A e^{-\gamma_{p} z} \Phi_{1}(z), \Phi_{2}(0)=0, \\
\frac{\partial \tilde{\Phi}_{3}\left(z, z_{l}\right)}{\partial z}= & 0, z \geq z_{l}, \\
& \tilde{\Phi}_{3}\left(z_{l}, z_{l}\right)=-i \sqrt{2 \gamma_{s}} \Phi_{1}\left(z_{l}\right), \\
\frac{\partial \tilde{\Phi}_{4}\left(z, z_{l}\right)}{\partial z}= & -\left(i\left(\beta_{s}+\beta_{i}\right)+\gamma_{s}+\gamma_{i}\right) \tilde{\Phi}_{4}\left(z, z_{l}\right), \\
z \geq & z_{l}, \quad \tilde{\Phi}_{4}\left(z_{l}, z_{l}\right)=-i \sqrt{2 \gamma_{s}} \Phi_{2}\left(z_{l}\right), \\
\frac{\partial \tilde{\Phi}_{5}\left(z, z_{l}\right)}{\partial z}= & -\left(i 2 \beta_{s}+2 \gamma_{s}\right) \tilde{\Phi}_{5}\left(z, z_{l}\right), \\
z \geq & z_{l}, \quad \tilde{\Phi}_{5}\left(z_{l}, z_{l}\right)=-i \sqrt{2 \gamma_{i}} \Phi_{2}\left(z_{l}\right) .
\end{aligned}
$$

Note that the initial conditions at $z=0$ correspond to one signal photon.

It is instructive to compare our approximate method with the exact model in the absence of losses, which has been extensively analyzed [10, 15-18]. Specifically, we employ the coupled Schrödinger equations in Fock space for photon-number amplitudes [24]. We observe that after interchanging the signal and idler photons in Eqs. (24),(25) of Ref. [24] and considering $N=1$ (difference between the number of signal and idler photons at the input), this model represents the dynamics of a single signal photon state, $\psi_{n=0}(z)$, and state of two signal and one idler photons, $\psi_{n=1}(z)$, and these equations coincide with our Eqs. (8),(9) up to a simple rescaling of the wavefunctions as $\Phi_{1}(z) \rightarrow \psi_{0}(z)$ and $\Phi_{2}(z) \rightarrow \psi_{1}(z) / \sqrt{2}$, and a change of notation of the normalized pump amplitude $A \rightarrow \gamma$. We note that the average signal photon number is found according to Eq. (38) of Ref. [24], and for a single signal photon input we have: $\left\langle n_{s}(z)\right\rangle=1+2|A|^{2}|b|^{-2}|\sinh (b z)|^{2}$, where $b=\sqrt{|A|^{2}-\left(\beta_{s}+\beta_{i}\right)^{2} / 4}$. Then, the limit of applicability of our approach corresponds to the regime where 
$\left\langle n_{s}(z)\right\rangle \ll 2$. This condition is satisfied if (i) $|A| z \ll 1$ close to phase-matching for $\left|\beta_{s}+\beta_{i}\right| \ll|A|$ and (ii) for all distances at large phase mismatches with $\left|\beta_{s}+\beta_{i}\right| \gg|A|$. In the regime of intermediate mismatches, the applicability condition can be verified numerically.

\section{ANALYSIS OF QUANTUM STATE EVOLUTION}

We note that Eqs. (8)-(9) are only coupled to each other, and thus can be solved separately. After that, it is straightforward to formulate solutions of Eqs. (10)-(12).
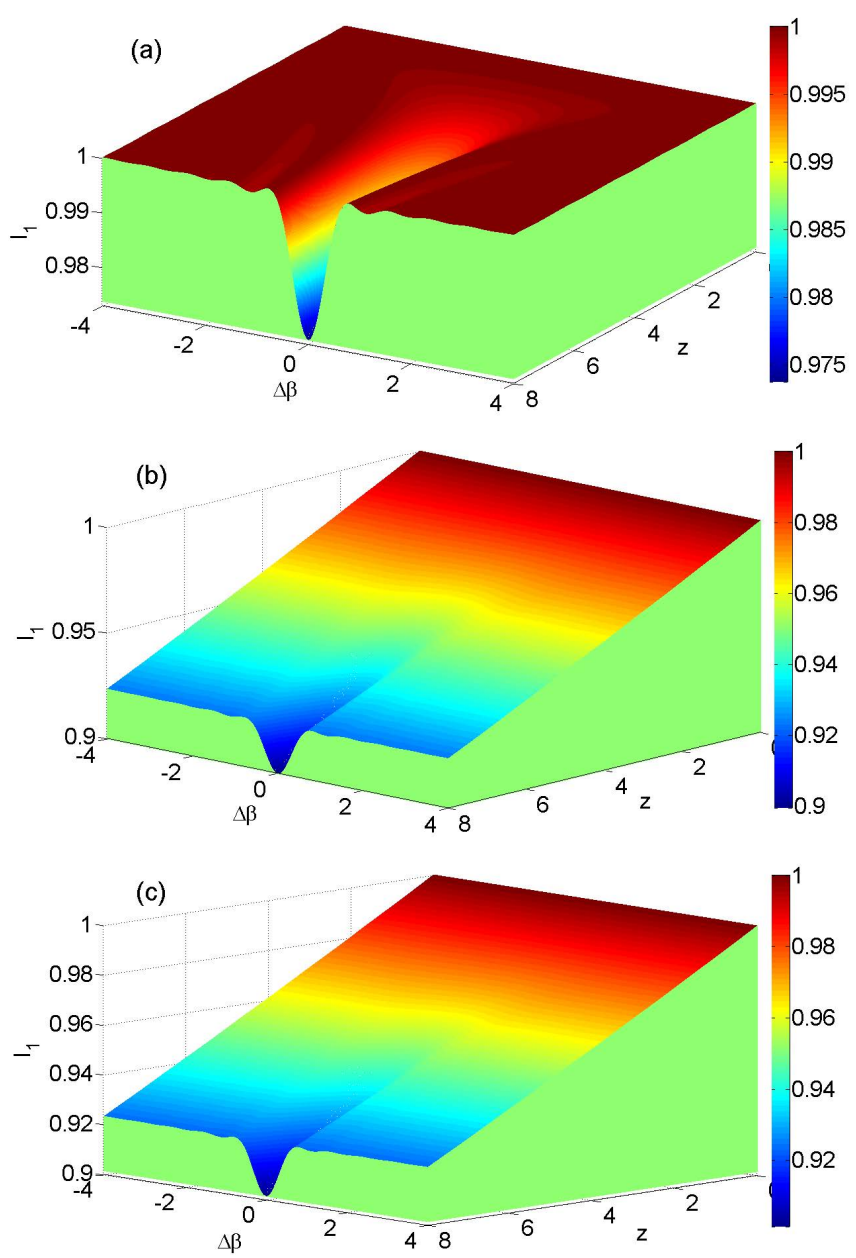

FIG. 2. Normalized number of individual signal photons $I_{1}(z)$ (without paired idler photons) vs. the phase mismatch $\Delta \beta$ and the propagation distance $z$ for $A=0.0144$, and different losses: (a) $\gamma_{p}=\gamma_{s}=\gamma_{i}=0$, (b) $\gamma_{p}=0, \gamma_{s}=\gamma_{i}=0.005$, (c) $\gamma_{p} / 2=\gamma_{s}=\gamma_{i}=0.005$.

Equations (8)-(9) can be solved analytically in the ab-
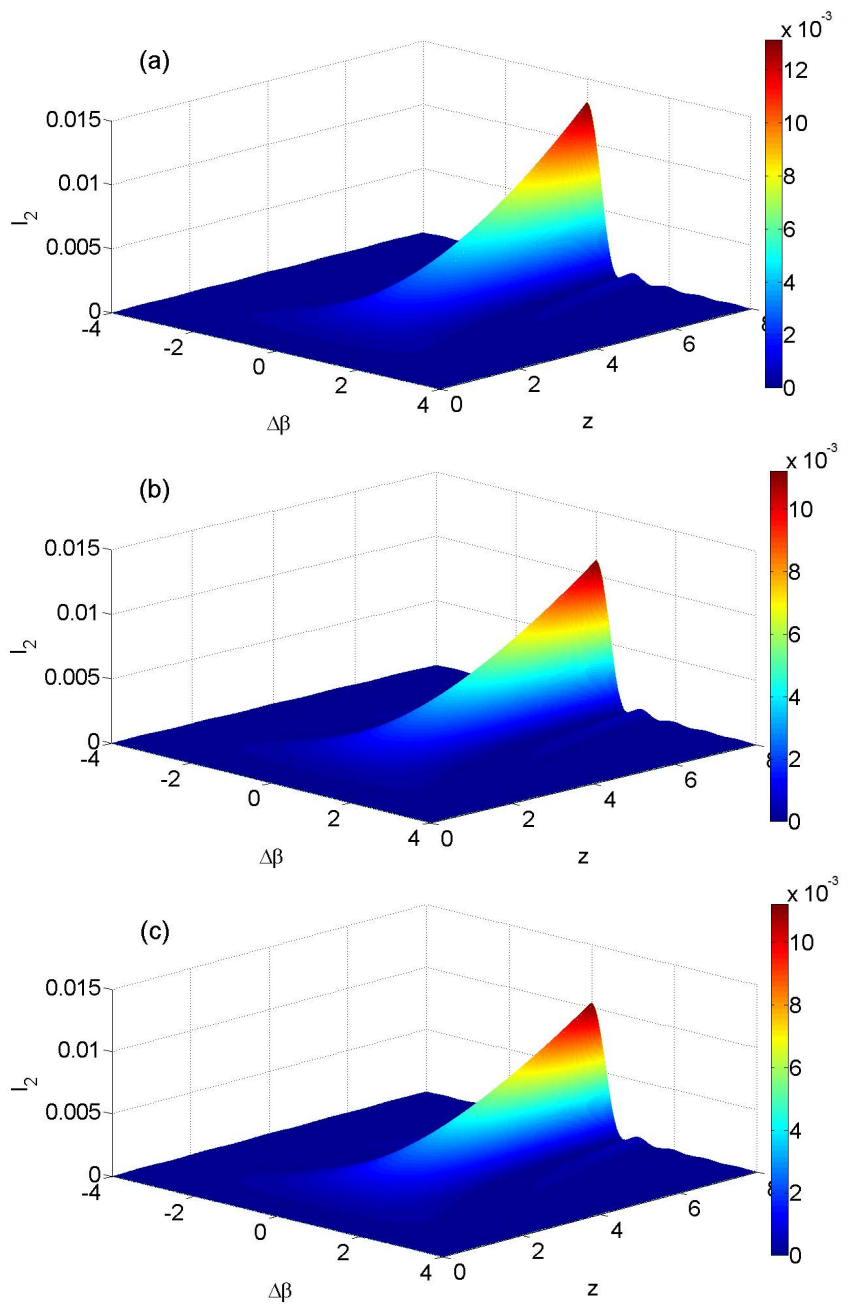

FIG. 3. Normalized number of photon triplets (two signal and one idler) $I_{2}(z)$. Notations and parameters are the same as in Fig. 2.

sence of pump losses, when $\gamma_{p}=0$ :

$$
\begin{aligned}
& \Phi_{1}(z)=e^{i k_{0} z}\left[\cos (\Delta k z)+\frac{\tilde{\beta}_{s}+\tilde{\beta}_{i}}{2 \Delta k} i \sin (\Delta k z)\right], \\
& \Phi_{2}(z)=-i \frac{A}{\Delta k} e^{i k_{0} z} \sin (\Delta k z)
\end{aligned}
$$

where we introduced the notations

$$
\begin{aligned}
k_{0} & =\frac{-\left(3 \tilde{\beta}_{s}+\tilde{\beta}_{i}\right)}{2}, \\
\tilde{\beta}_{s} & =\beta_{s}-i \gamma_{s}, \tilde{\beta}_{i}=\beta_{i}-i \gamma_{i}, \\
\Delta k & =\frac{1}{2} \sqrt{\left(\tilde{\beta}_{s}+\tilde{\beta}_{i}\right)^{2}+8 A^{2}} .
\end{aligned}
$$

When $\gamma_{p} \neq 0$, the Eqs. (8)-(9) can not be solved analytically in terms of elementary functions. In this regime, we obtain approximate solutions by perturbation theory, 
assuming that $A$ is small:

$$
\begin{aligned}
\Phi_{1}(z)= & e^{-i \tilde{\beta_{s}} z} \\
\Phi_{2}(z)= & -z i A \operatorname{sinc}\left[\frac{z}{2}\left(\tilde{\beta}_{s}+\tilde{\beta}_{i}+i \gamma_{p}\right)\right] \\
& \times e^{i k_{0} z} e^{-\gamma_{p} z / 2} .
\end{aligned}
$$

It can be checked that solutions in Eqs. (13)-(14) and Eqs. (18)-(19) coincide for $\gamma_{p}=0$ and $|A| \ll 1$.

The squared modulus of the wavefunction amplitudes provide the expectation values of the photon counts, which can be detected experimentally. Specifically, $I_{1}(z)=\left|\Phi_{1}(z)\right|^{2}$ is equal to the average number of signal photons without paired idler photons which can be detected at the coordinate $\mathrm{z}$, per unit time. The value of $I_{2}(z)=\left|\Phi_{2}(z)\right|^{2}$ is equal to the average number of sets of three photons (two signal and one idler) at coordinate $\mathrm{z}$ per unit time.

We now analyze in detail the solution in Eq. (19), describing the state of two signal and one idler photons, which is obtained through nonlinear parametric amplification of an input signal photon. The probability of this state can be expressed as

$$
\begin{aligned}
I_{2}(z) & =\left|\Phi_{2}(z)\right|^{2}=\mu\left(\cosh \left(z \Delta_{\gamma}\right)-\cos (z \Delta \beta)\right) e^{-\gamma_{p} z}(20) \\
\Delta_{\gamma} & =\gamma_{p}-\gamma_{i}-\gamma_{s} ; \mu=\frac{2 A^{2}}{(\Delta \beta)^{2}+\left(\Delta_{\gamma}\right)^{2}} .
\end{aligned}
$$

For $\gamma_{p}, \gamma_{i}, \gamma_{s} \ll z^{-1}$, we can approximately determine the minimum and maximum values of $I_{2}(z)$, taking into account that $\cosh \left(z \Delta_{\gamma}\right)$ is a slowly varying function:

$$
\begin{aligned}
& \min I_{2}(z) \approx\left(2 \pi N \Delta_{\gamma}\right)^{2} \frac{A^{2}}{\Delta \beta^{4}}, z_{\min } \approx \frac{2 \pi N}{\Delta \beta} \\
& \max I_{2}(z) \approx 4 \frac{A^{2}}{\Delta \beta^{2}} ; z_{\max } \approx \frac{2 \pi\left(N+\frac{1}{2}\right)}{\Delta \beta}
\end{aligned}
$$

where $N=0,1,2, \ldots$.

Note that in the case when the losses are such that $\Delta_{\gamma}=0,\left|\Phi_{2}(z)\right|^{2}$ reaches exactly zero, which can be also established directly from Eq. (21) even when losses are not weak. However for different losses when $\Delta_{\gamma} \neq 0$, the minimum value is non-zero and increases quadratically with the number of oscillations $N$. On the other hand, for any weak losses, the maximum value is only dependent on the pump amplitude and the phase mismatch.

Next, we determine the total number of signal and idler pairs or paired signal photons as

$$
\begin{aligned}
P_{4}(z) & =\int_{0}^{z} d z_{l}\left|\tilde{\Phi}_{4}\left(z, z_{l}\right)\right|^{2}=2 \mu \gamma_{s} e^{-2\left(\gamma_{s}+\gamma_{i}\right) z} \\
& \times\left[\frac{\left(\sinh \left(\Delta_{\gamma} \mathrm{z}\right) \Delta_{\gamma}+\cosh \left(\Delta_{\gamma} \mathrm{z}\right) \gamma_{4}\right) \mathrm{e}^{-\gamma_{4} \mathrm{z}}-\gamma_{4}}{\left(\Delta_{\gamma}\right)^{2}-\gamma_{4}^{2}}\right. \\
& \left.+\frac{\left(-\sin (\Delta \beta \mathrm{z}) \Delta \beta+\cos (\Delta \beta \mathrm{z}) \gamma_{4}\right) \mathrm{e}^{-\gamma_{4} \mathrm{z}}-\gamma_{4}}{(\Delta \beta)^{2}+\gamma_{4}^{2}}\right](24) \\
P_{5}(z) & =\int_{0}^{z} d z_{l}\left|\tilde{\Phi}_{5}\left(z, z_{l}\right)\right|^{2}=2 \mu \gamma_{i} e^{-4 \gamma_{s} z} \\
& \times\left[\frac{\left(\sinh \left(\Delta_{\gamma} \mathrm{z}\right) \Delta_{\gamma}+\cosh \left(\Delta_{\gamma} \mathrm{z}\right) \gamma_{5}\right) \mathrm{e}^{-\gamma_{5} \mathrm{z}}-\gamma_{5}}{\left(\Delta_{\gamma}\right)^{2}-\gamma_{5}^{2}}\right. \\
& \left.+\frac{\left(-\sin (\Delta \beta \mathrm{z}) \Delta \beta+\cos (\Delta \beta \mathrm{z}) \gamma_{5}\right) \mathrm{e}^{-\gamma_{5} \mathrm{z}}-\gamma_{5}}{(\Delta \beta)^{2}+\gamma_{5}^{2}}\right](25)
\end{aligned}
$$

where $\gamma_{4}=\gamma_{p}-2\left(\gamma_{s}+\gamma_{i}\right)$ and $\gamma_{5}=\gamma_{p}-4 \gamma_{s}$. We now estimate the magnitude of these terms compared to $I_{2}(z)$ :

$$
\frac{P_{4}(z)}{I_{2}(z)}=\frac{2}{3} \gamma_{s} z+O\left(z^{2}\right), \frac{P_{5}(z)}{I_{2}(z)}=\frac{2}{3} \gamma_{i} z+O\left(z^{2}\right),
$$

and see that these are indeed higher-order corrections, confirming the validity of our approach for $\gamma_{s, i} z \ll 1$.

Next, we perform numerical simulations to directly solve Eqs. (8)-(9), without resorting to analytial approximations. We present results for the normalized pump value of $A=0.0144$, such that the applicability condition of our model equations is satisfied for distances up to $z \lesssim 10$. We note that Eqs. (8)-(12) are invariant under the transformations $A \rightarrow \rho A, z \rightarrow \rho^{-1} z, \beta_{p, s, i} \rightarrow \rho \beta_{p, s, i}$, $\gamma_{p, s, i} \rightarrow \rho \gamma_{p, s, i}, \tilde{\Phi}_{3,4,5} \rightarrow \rho^{1 / 2} \tilde{\Phi}_{3,4,5}$ where $\rho$ is an arbitrary scaling coefficient. Accordingly, results can be simply rescaled for different pump amplitudes.

We present the dependencies of the single-signal and photon-triplet expectations $I_{1}$ and $I_{2}$ along the propagation direction $z$ for different phase mismatches $\Delta \beta$ in Figs. 2 and 3, respectively. In case without losses, the single-photon population only decreases due to the creation of photon triplets, which happens efficiently close to zero phase mismatch, see Figs. 2(a) and 3(a). In presence of losses, we observe an overall decrease of the signal photon counts, although creation of photon triplets can still be quite efficient, see Figs. 2(b,c) and 3(b,c).

We now consider in more detail the oscillations of photon state along the propagation direction in presence of the phase mismatch. We present in Figs. 4(a-c) the normalized number of photon triplets, consisting of two signal and one idler photons, as determined by the function $I_{2}(z)$, at its sequential local minima ( $N$ numbers the minima). Shown are the numerical results and approximate analytical predictions according to Eq. (22).

In the absence of loss, we see in Fig. 4(a) that the photon triplet state completely vanishes periodically, within the limits of numerical accuracy of the order of $10^{-10}$. If signal and idler photons exhibit loss, but there is no loss for the pump, then the photon triplet state does not vanish, and its minimum population grows quadratically 


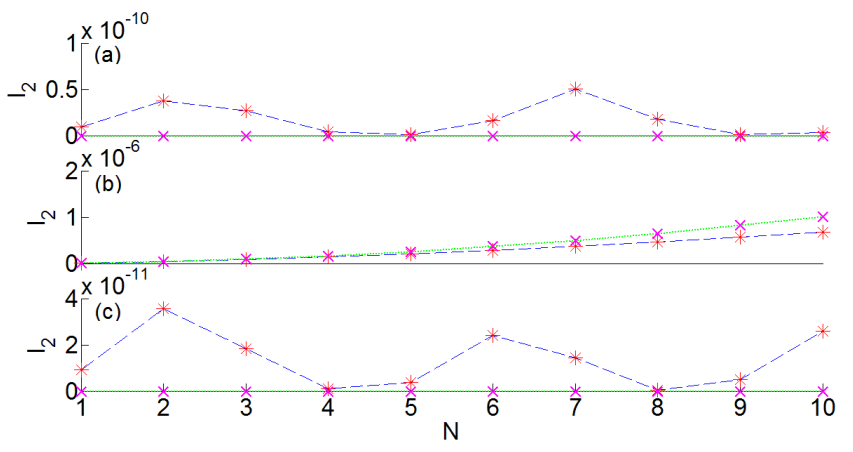

FIG. 4. Normalized number of photon triplets (two signal and one idler) at the sequential minima (number $N$ ) of $I_{2}(z)$. Shown are numerically calculated values (asterisks connect by blue dashed lines) and the approximate analytical results according to Eq. (22) (cross signs connect by green dots). For all the plots $\Delta \beta=3$, and the losses are: (a) $\gamma_{p}=\gamma_{s}=\gamma_{i}=0$, (b) $\gamma_{p}=0, \gamma_{s}=\gamma_{i}=0.005$, (c) $\gamma_{p} / 2=\gamma_{s}=\gamma_{i}=0.005$.

as predicted by Eq. (22) and observed in Fig. 4(b). However, when pump exhibits loss at half the rate of signal and idler photons, then photon triplet state again vanishes at minima up to numerical noise, see Fig. 4(c). This illustrates a nontrivial interplay between the losses of the pump and down-converted photons, where specific effects of loss can actually be compensated.

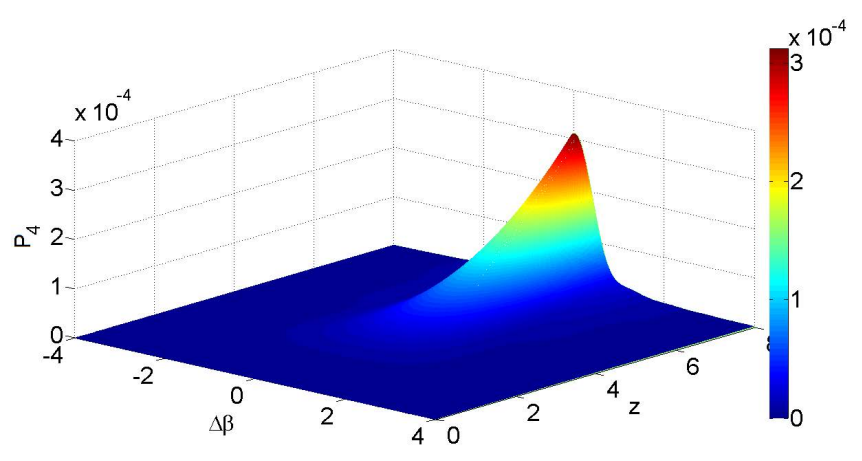

FIG. 5. The total number of signal and idler pairs $\left(P_{4}(z)\right)$ and paired signal photons $\left(P_{5}(z)\right)$. Notations and parameters are the same as in Fig. 2(b), and under such conditions $P_{4}(z) \equiv$ $P_{5}(z)$.

We also calculate the total number of signal and idler pairs $\left(P_{4}(z)\right)$ and paired signal photons $\left(P_{5}(z)\right)$. Characteristic results are presented in Fig. 5, which also agree closely with the analytical approximate solution in Eqs. (24),(25). We observe that these terms are generally smaller then triplet states $\left(I_{2}\right)$, c.f. Fig. 2(b). This further confirms the validity of our approach in the regime of small losses.

\section{CONCLUSION}

In conclusion, we have performed analytical and numerical analysis of the effect of linear losses on parametric amplification of a single photon, considering in detail single-photon and triple-photon outputs under different propagation distances and phase mismatches. In particular, we predict and confirm with simulations the effect of periodic suppression of three-photon states for a particular relation between the losses of signal and idler photons and the pump. Our method can be potentially extended to analyze the effect of losses on other regimes of nonlinear parametric interactions, including coherent single-photon conversion $[25,26]$ and cascaded parametric down-conversion [27].

\section{ACKNOWLEDGEMENTS}

This work was financially supported by the Government of Russian Federation (Grant 074-U01) and the Australian Research Council (Discovery Projects DP130100135 and DP160100619).

\section{Appendix A: Derivation of the coupled Schrödinger equations for photon number states}

In this Appendix we present a detailed derivation of Eqs. (8)-(12), using an approach formulated in Ref. [19].

We represent the photon state in the form of Eq. (6), and consider the input with only one signal photon:

$$
\begin{aligned}
\Phi_{1}(z=0) & =1, \\
\Phi_{2}(z=0) & =0, \\
\tilde{\Phi}_{3,4,5}^{(i)}\left(z=0, z_{l}\right) & =0 .
\end{aligned}
$$

Then, we introduce a notation $\tilde{A}(z)=A e^{-\gamma_{p} z}=$ $\kappa E_{p}\left(z, \omega_{p}\right)$ and substitute Eq. (6) into Eq. (7) and collect terms representing different photon states:

$$
\begin{aligned}
\frac{\partial \Phi_{1}(z)}{\partial z} & =-i\left[\beta_{s} \Phi_{1}(z)+2 \tilde{A}^{*} \Phi_{2}(z)\right. \\
& \left.+\frac{1}{2} \sqrt{2 \gamma_{s}} \tilde{\Phi}_{3}(z, z)\right] \\
\frac{\partial \Phi_{2}(z)}{\partial z} & =-i\left[\left(2 \beta_{s}+\beta_{i}\right) \Phi_{2}(z)+\tilde{A} \Phi_{1}(z)\right. \\
& \left.+\frac{1}{2} \sqrt{2 \gamma_{s}} \tilde{\Phi}_{4}(z, z)+\frac{1}{2} \sqrt{2 \gamma_{i}} \tilde{\Phi}_{5}(z, z)\right] .
\end{aligned}
$$

and 


$$
\begin{aligned}
\frac{\partial \tilde{\Phi}_{3}\left(z, z_{l}\right)}{\partial z} \Theta\left(z-z_{l}\right) & =-i \tilde{A}^{*} \tilde{\Phi}_{4}\left(z, z_{l}\right) \Theta\left(z-z_{l}\right)-i \sqrt{2 \gamma_{s}} \Phi_{1}(z) \delta\left(z-z_{l}\right)-\tilde{\Phi}_{3}(z, z) \delta\left(z-z_{l}\right), \\
\frac{\partial \tilde{\Phi}_{4}\left(z, z_{l}\right)}{\partial z} \Theta\left(z-z_{l}\right) & =-i\left[\beta_{s} \tilde{\Phi}_{4}\left(z, z_{l}\right)+\beta_{i} \tilde{\Phi}_{4}\left(z, z_{l}\right)+\tilde{A} \tilde{\Phi}_{3}\left(z, z_{l}\right)\right] \Theta\left(z-z_{l}\right) \\
& -\left[i 2 \sqrt{2 \gamma_{s}} \Phi_{2}(z)+\tilde{\Phi}_{4}(z, z)\right] \delta\left(z-z_{l}\right), \\
\frac{\partial \tilde{\Phi}_{5}\left(z, z_{l}\right)}{\partial z} \Theta\left(z-z_{l}\right) & =-i 2 \beta_{s} \tilde{\Phi}_{5}\left(z, z_{l}\right) \Theta\left(z-z_{l}\right)-i \sqrt{2 \gamma_{i}} \Phi_{2}(z) \delta\left(z-z_{l}\right)-\tilde{\Phi}_{5}(z, z) \delta\left(z-z_{l}\right),
\end{aligned}
$$

where $\Theta(z)$ is a Heaviside step function:

We also find the state where signal and idler photons are lost at the coordinates $z_{l_{s}}$ and $z_{l_{i}}$ respectively, and only one signal photon remains:

$$
0=2 \sqrt{2 \gamma_{s}} \tilde{\Phi}_{5}\left(z, z_{l_{i}}\right) \delta\left(z-z_{l_{s}}\right) \Theta\left(z-z_{l_{i}}\right)+\sqrt{2 \gamma_{i}} \tilde{\Phi}_{4}\left(z, z_{l_{s}}\right) \delta\left(z-z_{l_{i}}\right) \Theta\left(z-z_{l_{s}}\right) .
$$

We can solve Eq. (A7), taking into account the initial conditions:

$$
\begin{aligned}
& \tilde{\Phi}_{5}\left(z_{l_{s}}, z_{l_{i}}\right)=0, z_{l_{s}} \geq z_{l_{i}}, \\
& \tilde{\Phi}_{4}\left(z_{l_{i}}, z_{l_{s}}\right)=0, z_{l_{s}} \leq z_{l_{i}} .
\end{aligned}
$$

Next, we integrate Eqs. (A4)-(A6) for $z_{l}-\epsilon<z<z_{l}+\epsilon$ in the limit $\epsilon \rightarrow 0$ :

$$
\begin{gathered}
\tilde{\Phi}_{3}\left(z_{l}, z_{l}\right)=-i \sqrt{2 \gamma_{s}} \Phi_{1}\left(z_{l}\right), \tilde{\Phi}_{3}\left(z<z_{l}, z_{l}\right)=0 \\
\tilde{\Phi}_{4}\left(z_{l}, z_{l}\right)=-i 2 \sqrt{2 \gamma_{s}} \Phi_{2}\left(z_{l}\right), \tilde{\Phi}_{4}\left(z<z_{l}, z_{l}\right)=0 \\
\tilde{\Phi}_{5}\left(z_{l}, z_{l}\right)=-i \sqrt{2 \gamma_{i}} \Phi_{2}\left(z_{l}\right), \\
\tilde{\Phi}_{5}\left(z<z_{l}, z_{l}\right)=0 .
\end{gathered}
$$

We now substitute Eqs. (A8) and (A9) into Eqs. (A2)(A6):

$$
\begin{aligned}
\frac{\partial \Phi_{1}(z)}{\partial z}= & -\left(i \beta_{s}+\gamma_{s}\right) \Phi_{1}(z)-i 2 \tilde{A}^{*} \Phi_{2}(z), \\
\frac{\partial \Phi_{2}(z)}{\partial z}= & -\left(i\left(2 \beta_{s}+\beta_{i}\right)+2 \gamma_{s}+\gamma_{i}\right) \Phi_{2}(z) \\
& -i \tilde{A} \Phi_{1}(z), \\
\frac{\partial \tilde{\Phi}_{3}\left(z, z_{l}\right)}{\partial z}= & -i \tilde{A}^{*} \tilde{\Phi}_{4}\left(z, z_{l}\right), z \geq z_{l}, \\
\frac{\partial \tilde{\Phi}_{4}\left(z, z_{l}\right)}{\partial z}= & -\left(i\left(\beta_{s}+\beta_{i}\right)+\gamma_{s}+\gamma_{i}\right) \tilde{\Phi}_{4}\left(z, z_{l}\right) \\
& -i \tilde{A} \tilde{\Phi}_{3}\left(z, z_{l}\right), z \geq z_{l}, \\
\frac{\partial \tilde{\Phi}_{5}\left(z, z_{l}\right)}{\partial z}= & -\left(i 2 \beta_{s}+2 \gamma_{s}\right) \tilde{\Phi}_{5}\left(z, z_{l}\right), z \geq z_{l} .
\end{aligned}
$$

In Eqs. (A13) and (A14) we include losses through the coefficients $\gamma_{s, i}$. These coefficients emerge from the higher order terms of Eq. (6), which we took into account to properly account for losses: $\quad \int_{0}^{z} d z_{l_{s}} \int_{z_{l_{s}}}^{z} d z_{l_{i}} \tilde{\Phi}_{6}\left(z, z_{l_{s}}, z_{l_{i}}\right) b_{i}^{\dagger}\left(z_{l_{i}}\right) b_{s}^{\dagger}\left(z_{l_{s}}\right) a_{s}^{\dagger}|0\rangle$, $\int_{0}^{z} d z_{l_{s}} \int_{z_{l_{s}}}^{z} d z_{l_{s 2}} \tilde{\Phi}_{7}\left(z, z_{l_{s}}, z_{l_{s 2}}\right) b_{s}^{\dagger}\left(z_{l_{s}}\right) b_{s}^{\dagger}\left(z_{l_{s 2}}\right) a_{i}^{\dagger}|0\rangle$ $\int_{0}^{z} d z_{l_{i}} \int_{z_{l_{i}}}^{z} d z_{l_{s}} \tilde{\Phi}_{8}\left(z, z_{l_{s}}, z_{l_{i}}\right) b_{i}^{\dagger}\left(z_{l_{i}}\right) b_{s}^{\dagger}\left(z_{l_{s}}\right) a_{s}^{\dagger}|0\rangle$.

Next, we neglect the terms $\tilde{A}^{*} \tilde{\Phi}_{4}\left(z, z_{l}\right)$ and $\tilde{A} \tilde{\Phi}_{3}\left(z, z_{l}\right)$ in Eqs. (A12) and (A13), as they correspond to secondorder nonlinear amplification effects, which are assumed to be weak.
Finally, we obtain Eqs. (8)-(12).
[1] D. Bouwmeester, J. W. Pan, K. Mattle, M. Eibl, H. Weinfurter, and A. Zeilinger, Nature 390, 575 (1997).

[2] D. Boschi, S. Branca, F. De Martini, L. Hardy, and S. Popescu, Phys. Rev. Lett. 80, 1121 (1998).

[3] J. W. Pan, D. Bouwmeester, H. Weinfurter, and A. Zeilinger, Phys. Rev. Lett. 80, 3891 (1998).
[4] A. Furusawa, J. L. Sorensen, S. L. Braunstein, C. A. Fuchs, H. J. Kimble, and E. S. Polzik, Science 282, 706 (1998).

[5] A. K. Ekert, J. G. Rarity, P. R. Tapster, and G. M. Palma, Phys. Rev. Lett. 69, 1293 (1992).

[6] M. Giustina, A. Mech, S. Ramelow, B. Wittmann, 
J. Kofler, J. Beyer, A. Lita, B. Calkins, T. Gerrits, S. Nam, R. Ursin, and A. Zeilinger, Nature 497, 227 (2013).

[7] R. Fickler, M. Krenn, R. Lapkiewicz, S. Ramelow, and A. Zeilinger, Sci. Rep. 3, 1914 (2013).

[8] S. Tanzilli, H. De Riedmatten, W. Tittel, H. Zbinden, P. Baldi, M. De Micheli, D. B. Ostrowsky, and N. Gisin, Electron. Lett. 37, 26 (2001).

[9] K. Banaszek, A. B. U'Ren, and I. A. Walmsley, Opt. Lett. 26, 1367 (2001).

[10] F. De Martini and F. Sciarrino, Prog. Quantum Electron. 29, 165 (2005).

[11] Q. Zhang, X. P. Xie, H. Takesue, S. W. Nam, C. Langrock, M. M. Fejer, and Y. Yamamoto, Opt. Express 15, 10288 (2007).

[12] Q. Zhang, H. Takesue, C. Langrock, X. P. Xie, M. M. Fejer, and Y. Yamamoto, Jpn. J. Appl. Phys. 49, 064401 (2010).

[13] A. Eckstein and C. Silberhorn, Opt. Lett. 33, 1825 (2008).

[14] M. F. Saleh, G. Di Giuseppe, B. E. A. Saleh, and M. C. Teich, IEEE Photonics J. 2, 736 (2010).

[15] M. K. Olsen, L. I. Plimak, and A. Z. Khoury, Opt. Commun. 215, 101 (2003).

[16] X. X. Xu and H. C. Yuan, Int. J. Theor. Phys. 53, 1601 (2014).
[17] R. Ghobadi, A. Lvovsky, and C. Simon, Phys. Rev. Lett. 110, 170406 (2013).

[18] C. Vitelli, L. Toffoli, F. Sciarrino, and F. D. Martini, in Personal Satellite Services (PSATS), Lecture Notes of the Institute for Computer Sciences, Social Informatics and Telecommunications Engineering, Vol. 43, edited by K. Sithamparanathan, M. Marchese, M. Ruggieri, and I. Bisio (Springer-Verlag, Berlin, 2010) pp. 330-339.

[19] D. A. Antonosyan, A. S. Solntsev, and A. A. Sukhorukov, Phys. Rev. A 90, 043845 (2014).

[20] L. G. Helt, M. J. Steel, and J. E. Sipe, New J. Phys. 17, 013055 (2015).

[21] N. A. Silva and A. N. Pinto, IEEE J. Quantum Electron. 48, 1380 (2012).

[22] N. A. Silva and A. N. Pinto, J. Lightwave Technol. 31, 1309 (2013).

[23] R. Kruse, F. Katzschmann, A. Christ, A. Schreiber, S. Wilhelm, K. Laiho, A. Gabris, C. S. Hamilton, I. Jex, and C. Silberhorn, New J. Phys. 15, 083046 (2013).

[24] A. A. Sukhorukov, A. S. Solntsev, and J. E. Sipe, Phys. Rev. A 87, 053823 (2013).

[25] N. K. Langford, S. Ramelow, R. Prevedel, W. J. Munro, G. J. Milburn, and A. Zeilinger, Nature 478, 360 (2011).

[26] D. A. Antonosyan, A. S. Solntsev, and A. A. Sukhorukov, Opt. Commun. 327, 22 (2014).

[27] H. Hubel, D. R. Hamel, A. Fedrizzi, S. Ramelow, K. J. Resch, and T. Jennewein, Nature 466, 601 (2010). 